\section{Investigación original}

dx.doi.org/10.35366/CMA201B

\title{
Bloqueo regional intraarticular con anestésicos locales en cirugía artroscópica de rodilla
}

\author{
Regional intra-articular block with local \\ anesthetics in arthroscopic knee surgery
}

\author{
Prof. Dra. Idoris Cordero-Escobar, ${ }^{*}$ \\ Prof. Dra. Araceli Chico-Capote, ${ }^{\ddagger}$ Dr. José Escobar-Andarade, ${ }^{\S}$ \\ Dr. Miguel H Estévez-Chico, ${ }^{\S}$ Dra. Isabel Mora-Díaz"
}

\begin{abstract}
RESUMEN. Introducción: La técnica anestésica de elección para la artroscopía está determinada por los requerimientos quirúrgicos intraoperatorios y pretende optimizar la recuperación, el control del dolor postoperatorio y facilitar el alta precoz. Objetivo: Evaluar los resultados del bloqueo regional intraarticular con anestésicos locales en la cirugía artroscópica de rodilla. Material y métodos: Se realizó un estudio observacional, descriptivo, de corte transversal para evaluar los resultados del bloqueo regional intraarticular con anestésicos locales en la cirugía artroscópica de rodilla, en el Hospital Clínico Quirúrgico «Hermanos Ameijeiras», en el período comprendido entre febrero de 2013 y febrero de 2016. El estudio incluyó un total de 120 pacientes, distribuidos en dos grupos de 60 cada uno. Grupo AS: se utilizó anestesia subaracnoidea y Grupo Al: se realizó anestesia intraarticular con anestesia local. Resultados: El grupo etario más frecuente fue entre 60-69 años, $28.3 \%$ en el Grupo AS y $35.0 \%$ en el Grupo IA, sin diferencias significativas ( $p$ $=0.683)$. Predominó el sexo femenino sobre el masculino (42/18 vs $44 / 16)$, sin diferencias significativas $(p=0.839)$. Predominó el ASA I, en 56.7 y $43.3 \%$, según técnica anestésica, sin diferencias significativas $(p=0.092)$. La rodilla derecha predominó sobre la izquierda. La entidad nosológica más frecuente fue la plica presente en $58.3 \%$ del Grupo AS y $60 \%$ en el Grupo IA $(p=0.408)$. La osteoartritis de rodilla, según grupos, se presentó en $46.7 \%$ en el grupo AS y en $50.0 \%$ del grupo Al. El dolor postoperatorio estuvo presente en $21.7 \%$ del grupo AS y en $10 \%$ en el Grupo IA. Existieron diferencias significativas entre grupos $(p=0.0114)$. Las complicaciones según grupos, se presentaron en el doble de pacientes del grupo AS $(30 \%)$ que en el Grupo IA (15\%) con diferencias significativas $(p=0.049)$. Conclusiones: Los resultados del bloqueo regional intraarticular con anestésicos locales en la cirugía artroscópica de rodilla constituye una técnica anestésica fácil de realizar y que brinda seguridad al paciente.
\end{abstract}

ABSTRACT. Introduction: The anesthetic technique of choice for arthroscopy is determined by the intraoperative surgical requirements and aims to optimize recovery, postoperative pain control and facilitate early discharge. Objective: To evaluate the results of intra-articular regional block with local anesthetics in arthroscopic knee surgery. Material and methods: An observational, descriptive, cross-sectional study was conducted to evaluate the results of the intraarticular regional block with local anesthetics in arthroscopic knee surgery at the «Hermanos Ameijeiras» Clinical Surgical Hospital, in the period between February 2013 and February 2016. The study included a total of 120 patients, distributed in two groups of 60 each. Group AS: subarachnoid anesthesia and Group Al: intra-articular regional block with local anesthetics. Results: The most frequent age group was between $60-69$ years, $28.3 \%$ in group $S A$ and $35.0 \%$ in group $I A$, without significant differences $(p=0.683)$. Female sex predominated over male $(42 / 18 \mathrm{vs} 44 / 16)$, without significant differences $(p=0.839)$. ASA I predominated, in $56.7 \%$ and $43.3 \%$, according to anesthetic technique, without significant differences $(p=0.092)$. The right knee predominated over the left. The most frequent nosological entity was the escrow present in $58.3 \%$ of the AS group and $60 \%$ in the group IA ( $p=0.408)$. Osteoarthritis of the knee, according to groups, was presented in $46.7 \%$ in the AS group and $50.0 \%$ in the Al group. Postoperative pain was present in $21.7 \%$ of the AS group and $10 \%$ in the Group IA. There were significant differences between groups $(p=0.0114)$. Complications according to groups occurred in twice as many patients in the AS group (30.0\%) as in group IA (15.0\%) with significant differences $(p=0.049)$. Conclusions: The results of intra-articular regional block with local anesthetics in arthroscopic knee surgery is an anesthetic technique that is easy to perform and provides patient safety.
Anestesiologla

Enero-Marzo 2020

Vol. 43. No. 1. pp 8-15

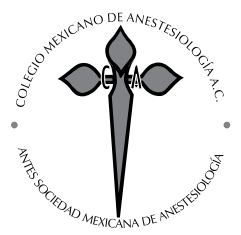

* Especialista en Anestesiología y Reanimación. MD, PhD.

Profesora Titular.

‡ Especialista en Reumatología. MD, PhD. Profesora Titular.

$\S$ Especialista en Anestesiología y Reanimación. MD.

" Especialista en Bioestadística. MD MSc. en Urgencias Médicas. Profesora Asistente. Servicio de Investigaciones.

Hospital Hermanos Ameijeiras. La Habana, Cuba.

Palabras clave: Artroscopía de rodilla, anestesia intraarticular, dolor postoperatorio, complicaciones.

Keywords: Knee arthroscopy, intra-articular anesthesia, postoperative pain, complications.

Solicitud de sobretiros:

Prof. Dra. Idoris Cordero Escobar

San Lázaro 701 entre Belascoain y Marques González, La Habana, Cuba. CP 10300

Recibido para publicación: 21-02-2019

Aceptado para publicación: 02-04-2019 


\section{INTRODUCCIÓN}

L a artroscopía es un método mínimamente invasivo para $\checkmark$ diagnosticar y tratar problemas articulares. Los avances tecnológicos en el campo de la artroscopía han hecho posible que cada vez se produzcan monitores de alta definición y cámaras de alta resolución para facilitar el proceder diagnóstico y terapéutico. Éstas y otras mejoras han transformado la artroscopía en una herramienta eficaz para tratar los problemas de rodilla.

Mondino ${ }^{(1)}$ publicó que los primeros procedimientos biopsicoartroscópicos con anestesia local se difundieron a finales de la década de los 60. Este autor refirió que McGinty y Matza desarrollaron la anestesia intraarticular de rodilla (AIAR) mediante la administración de bupivacaína al líquido de lavado; pero no la aconsejaron para método quirúrgico por el dolor y la desagradable sensación que se provocaba al traccionar o cortar, o ambos, el menisco. A finales de la década de los 80 y principio de los 90 aparecieron artículos que modificaron las técnicas, el tipo de anestésicos y volúmenes empleados, con el uso de vasoconstrictores ${ }^{(2-5)}$.

La artroscopía se ha convertido en un proceder extremadamente importante para el ortopédico y el reumatólogo, tanto en el diagnóstico como en los procedimientos quirúrgicos de las afecciones de la rodilla ${ }^{(2,3)}$. Este procedimiento puede realizarse con diferentes técnicas anestésicas: local, regional o general ${ }^{(6)}$.

Las técnicas anestésicas subaracnoidea o epidural se han utilizado de forma sistemática para la cirugía artroscópica de rodilla en España ${ }^{(4)}$. Dichas técnicas proporcionan un bloqueo completo sensitivo y motor de la extremidad inferior. Todos los que realizan esta técnica señalan las ventajas de la anestesia regional o general en relación con las comodidades para el cirujano o en relación con los niveles de analgesia que se obtienen; pero también reconocen sus desventajas relacionadas, fundamentalmente, con los riesgos para el paciente y las molestias de su recuperación como dolor lumbar, retención urinaria, cefaleas postpunción, entre otras. Aunque estas complicaciones no son tan frecuentes, hay que tener presente que existe una alternativa válida que es la $\operatorname{AIAR}^{(5,6)}$.

La mayoría de los autores ${ }^{(2-5)}$, señalaron que la técnica anestésica de elección para este procedimiento está determinada por los requerimientos quirúrgicos intraoperatorios, ya que se pretende optimizar la recuperación rápida del paciente con el control del dolor postoperatorio, de los eventos eméticos y facilitar el alta precoz ${ }^{(4,7-12)}$.

Los motivos que pudieron llevar a los anestesiólogos a elegir una técnica neuroaxial para la artroscopía de rodilla son varios, entre los cuales destacan la facilidad y la rapidez de ejecución de la técnica, los mínimos requerimientos en cuanto a los cuidados en el mantenimiento de la misma y la amplia experiencia adquirida en su rutina diaria. Es llamativo que la elección de la anestesia subaracnoidea es independiente del tiempo estimado del procedimiento quirúrgico, cuya duración fue inferior a una hora en casi $80 \%$ de los casos y de media hora en $20 \%{ }^{(13-25)}$.

Según la Sociedad Ortopédica Americana para la Medicina del Deporte, el empleo de anestésicos locales y/o morfina constituyen una estrategia anestésica y analgésica postoperatoria eficaz y satisfactoria, con la cual se realiza en todo el mundo anualmente más de cuatro millones de artroscopías de rodilla ${ }^{(24-27)}$.

Desde que se realiza la artroscopía de rodilla con anestesia local ${ }^{(3)}$, el resto de las técnicas anestésicas se han utilizado menos, pues el control del dolor intra y postoperatorio son suficientes y las complicaciones escasas ${ }^{(28)}$.

Constituye el objetivo de esta investigación evaluar los resultados del bloqueo regional intraarticular con anestésicos locales en la cirugía artroscópica de rodilla.

\section{MATERIAL Y MÉTODOS}

Se realizó un estudio observacional, descriptivo, de corte transversal con el propósito de evaluar los resultados del bloqueo regional intraarticular con anestésicos locales en la cirugía artroscópica de rodilla. El estudio se realizó en el Hospital Clínico Quirúrgico «Hermanos Ameijeiras», en el período comprendido entre febrero de 2013 y febrero de 2016.

Se consideraron los siguientes requisitos de inclusiónexclusión.

- Criterios de inclusión: pacientes entre 18 y 70 años, ASA I-III, de forma electiva que requieran anestesia para cualquier procedimiento quirúrgico de la rodilla mediante artroscopía.

- Criterios de exclusión: pacientes aprehensivos o poco colaboradores. Con antecedentes de alergia a los anestésicos locales tipo amida (lidocaína y bupivacaína), determinada por referencia de los pacientes en intervenciones anteriores. Con tumefacción y dolor de importancia. Con rango de movilidad menor de $90 \%$ y portadores de sinovitis.

Muestra: todos los pacientes que cumplieron con los criterios de selección en el período comprendido de febrero de 2013 hasta febrero de 2016.

Los pacientes fueron asignados de forma aleatoria en dos grupos de estudio según la técnica anestésica a utilizar:

- Grupo AS: se utilizó anestesia subaracnoidea.

- Grupo AI: se realizó anestesia intraarticular con anestesia local.

A todos los pacientes, se les solicitó el consentimiento informado por escrito. 


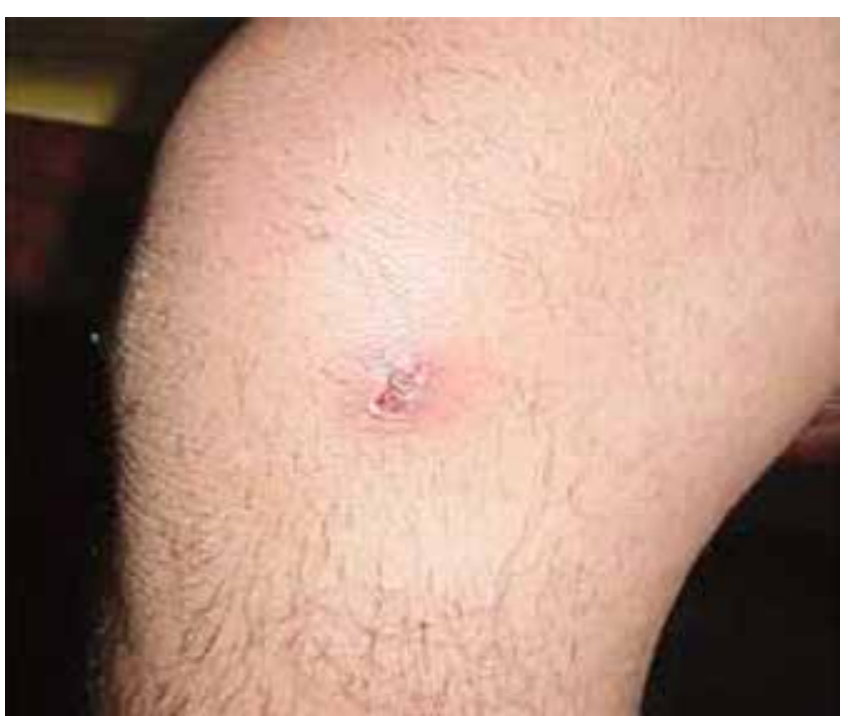

Figura 1: Infiltración local de la piel.

Procedimiento anestésico: en todos los casos se realizó igual medicación preoperatoria con midazolam $3 \mu \mathrm{g} / \mathrm{kg} \mathrm{IV}$.

Se evaluaron los signos vitales con un monitor modelo NIHON KOHDEN para la vigilancia de la frecuencia cardíaca (FC), la presión arterial sistólica (PAS) y diastólica (PAD), el trazado electrocardiográfico (ECG) y la pulsioximetría $\left(\mathrm{SatO}_{2}\right)$.

\section{Técnica anestésica}

Grupo AS. Previa asepsia y antisepsia se seleccionó el espacio interespinoso más amplio y se higienizó la región sobre la espina del sacro y las crestas ilíacas, todo exceso de antiséptico se retiró con tiempo suficiente para que actuara. Se colocaron los campos, de forma tal que suministraron una visión amplia de la columna lumbar, desde T12 a S1, y lateralmente se incluyó el área de los músculos cuadrados lumbares. Se infiltró un habón epidérmico con lidocaína al $1 \%$ contenido en una jeringa de $2 \mathrm{~mL}$. Se seleccionó una aguja subaracnoidea $25 \mathrm{~g}$. Se colocó el estilete en un ángulo menor a $5^{\circ}$ con el bisel paralelo al eje longitudinal de la columna. Se retiró el estilete para observar el flujo del líquido raquídeo. Se conectó a la aguja subaracnoidea una jeringa Luer Lock de $5 \mathrm{~mL}$, con una mezcla anestésica, lidocaína al 5\%, $1 \mathrm{~mL}$ (50 mg). En nuestro país, por razones del bloqueo económico, sólo tenemos lidocaína y bupivacaína, no se presentó neurotoxicidad. Después, se aspiró una pequeña cantidad de líquido raquídeo para definir si la aguja estaba en el sitio correcto y se inyectó la solución anestésica, se retiró la aguja y el introductor al mismo tiempo. Se esperó 15 minutos en posición sentada para la fijación del agente anestésico y por último se comprobó el nivel anestésico.
Grupo IA. Con el paciente en quirófano se procedió previa asepsia y antisepsia de la región. Luego, con la rodilla en flexión, se procedió a la infiltración de la solución del anestésico local.

Se realizó habón cutáneo en el cuadrante anteroexterno (Figura 1) y se administró $5 \mathrm{~mL}$ de lidocaína al $2 \%$ con epinefrina dos microgotas al $1 \times 5,200,000$.

Se llevó a cabo bloqueo a nivel del cuadrante antero interno (Figura 2). Se infiltró piel y se evitó piel de naranja, tejido celular subcutáneo y cápsula articular, extendiendo por la interlinea interna para bloquear el nervio infrapatelar y el ligamento lateral interno.

Posteriormente, con la rodilla en extensión, se procedió a la infiltración intraarticular (Figura 3) en el ángulo superoexterno de la rótula, asegurando que el extremo de la aguja (40/8) estuviera libre dentro de la articulación y no en el tejido sinovial (Figura 4); se colocaron de 10 a $15 \mathrm{~mL}$ de lidocaína al $2 \%$ con epinefrina.

Asimismo, se reservaron $2 \mathrm{~mL}$ de bupivacaína para que al final de la cirugía se pudiese prolongar la analgesia postoperatoria.

En caso que el paciente presentara hemartrosis/hidrartrosis, previamente se procedió a evacuar la articulación.

El dolor en el transoperatorio se evaluó por ausencia de dolor o molestias durante el procedimiento quirúrgico.
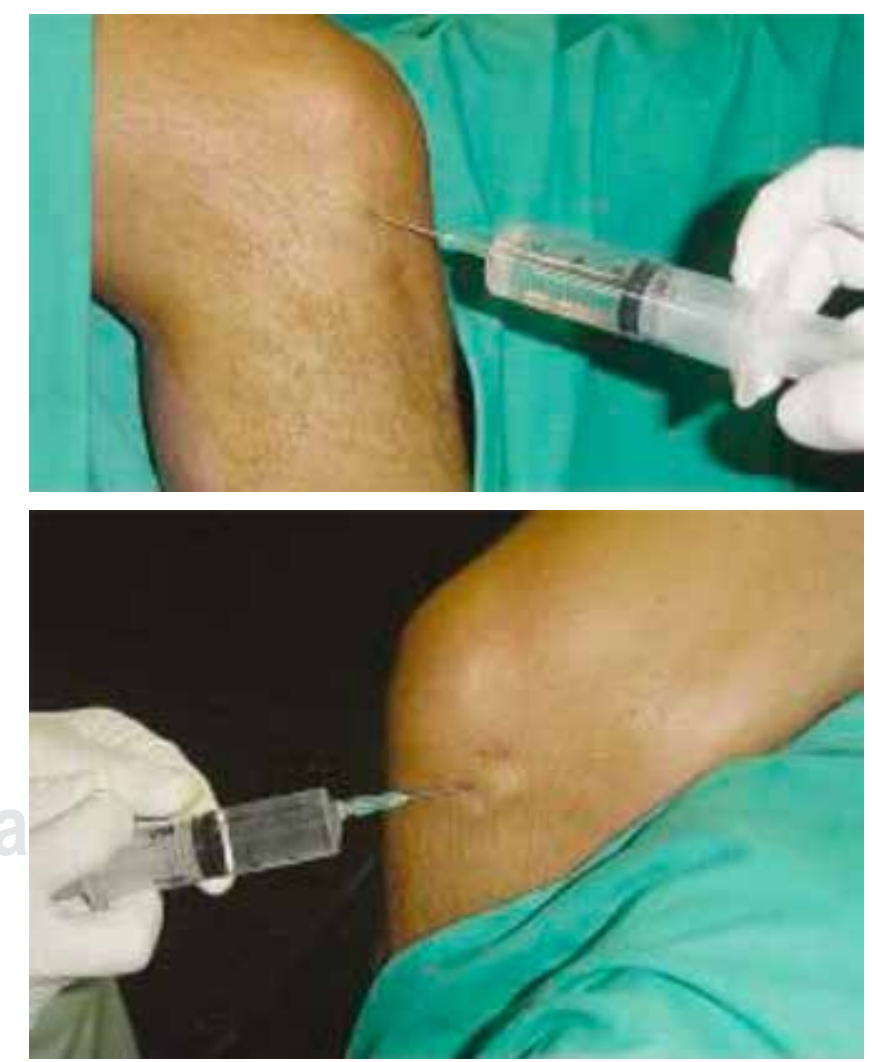

Figura 2: Técnica anestésica. 


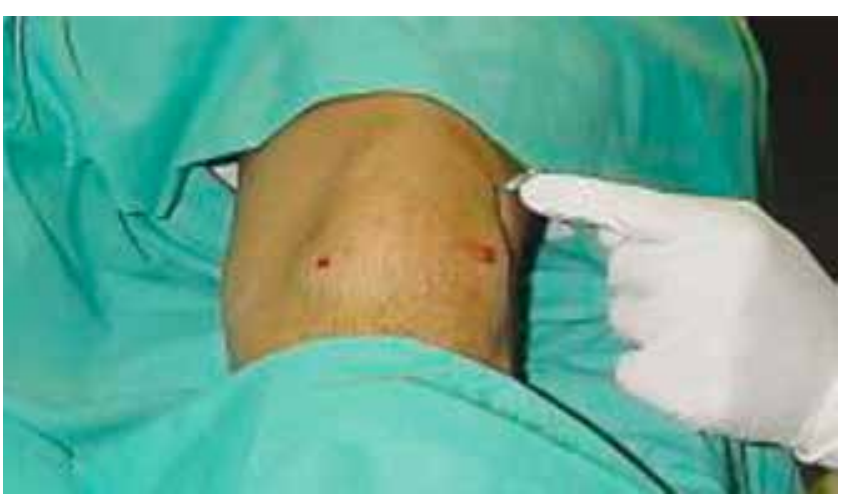

Figura 3: Infiltración intraarticular.

Analgesia postoperatoria. Con objeto de estandarizar la analgesia postoperatoria, los pacientes fueron provistos de 50 mg de diclofenaco intramuscular.

En todos los pacientes se realizó igual medicación preoperatoriamente con midazolam $3 \mu \mathrm{g} / \mathrm{kg}$ IV y no fue necesario analgésicos intraoperatorios.

La información se recogió a través de una hoja de recolección de datos provenientes de la historia clínica individual de anestesia.

El dolor intraoperatorio y postoperatorio se evaluó según escala visual análoga entre 0 y 10 , donde 0 es ausencia de dolor y 10 es dolor insoportable. Se clasificó de acuerdo al resultado en: leve de 0 a 3, moderada de 4 a 7 e intenso de 7 a 10.

Análisis estadístico. Para el procesamiento de los datos se utilizó una base de datos en Excel con el empleo del sistema computarizado SPSS 16.0. Para cumplimentar los objetivos propuestos se analizaron las variables seleccionadas mediante el cálculo de medidas de resumen para variables cualitativas y cuantitativas (números absolutos y porcentajes, media y desviación estándar). Para la comparación entre grupos se utilizó la prueba estadística chi-cuadrado $\left(\chi^{2}\right)$ de Pearson como método de análisis para evaluar la asociación entre variables cualitativas y la T Student para variables cuantitativas. Las diferencias fueron consideradas estadísticamente significativas cuando el valor de p sea menor que 0.05 .

Seguridad y ética. El protocolo de investigación se analizó y aprobó por los profesores del Servicio de Anestesiología y Reanimación del Hospital y la Comisión Científica de la Institución. Se tuvieron en cuenta todos los aspectos de seguridad, así como los aspectos éticos que caracterizan a toda investigación clínica.

\section{RESULTADOS}

Se estudiaron un total de 120 pacientes, distribuidos en 60 por cada grupo. La media de edad con mayor frecuencia fue el grupo de 60-69 años, 28.3\% en el Grupo AS y 35.0\% en el
Grupo IA. El sexo femenino predominó sobre el masculino (42/18 versus 44/16) en ambas técnicas anestésicas. El estado físico ASA I se presentó en 56.7 y 43.3\%, según grupos, sin diferencias significativas $(\mathrm{p}=0.092)$. El sobrepeso ligero se observó con mayor frecuencia en el Grupo AS (46.7\%) en comparación con el Grupo IA (38.3\%). No existieron diferencias significativas entre ambos grupos en ninguna de las variables señaladas.

En la Tabla 1 se muestra predominio de las afecciones en la rodilla derecha sobre la izquierda. La relación del Grupo AS sobre la IA fue: 42/18 y 35/25, 58.3\%, según la técnica anestésica empleada. La afección más frecuente fue la plica, la cual se presentó en 58.3\% del Grupo AS y 60\% en el Grupo IA $(p=0.408)$. No existieron diferencias entre grupos.

Se demostró la presencia o no de osteoartritis de rodilla, según grupos. No se presentó en $46.7 \%$ en el Grupo AS y en $50.0 \%$ del Grupo IA. Fue ligera en $6.7 \%$ la AS y $8.3 \%$ en la IA. Moderada entre 3.3\% del Grupo AS y 1.7\% del Grupo IA, mientras que fue severa en $43.3 \%$ del AS y $40.0 \%$ del IA (Tabla 2).

La evaluación del bloqueo regional con anestésicos locales, según presencia de dolor, se muestra en la Tabla 3. Se observa que en ambos grupos el dolor postoperatorio estuvo presente en $21.7 \%$ del Grupo AS y en 10\% del IA. A las seis horas del postoperatorio, el dolor se presentó en 16.7\% del Grupo AS y sólo en $1.7 \%$ del Grupo IA.

Según la escala análoga visual, la media del dolor en el intraoperatorio fue de 0 a 3 (leve). En el postoperatorio sólo un paciente del Grupo IA presentó dolor de 0 a 3 (leve) a las 12 horas, mientras que en el Grupo AS cinco pacientes presentaron dolor moderado (media de 7) a las seis y 12 horas. Existieron diferencias significativas entre grupos $(p=0.0114)$.

La presencia de complicaciones según grupos se indica en la Tabla 4. En el Grupo AS, 30\% de los casos presentaron

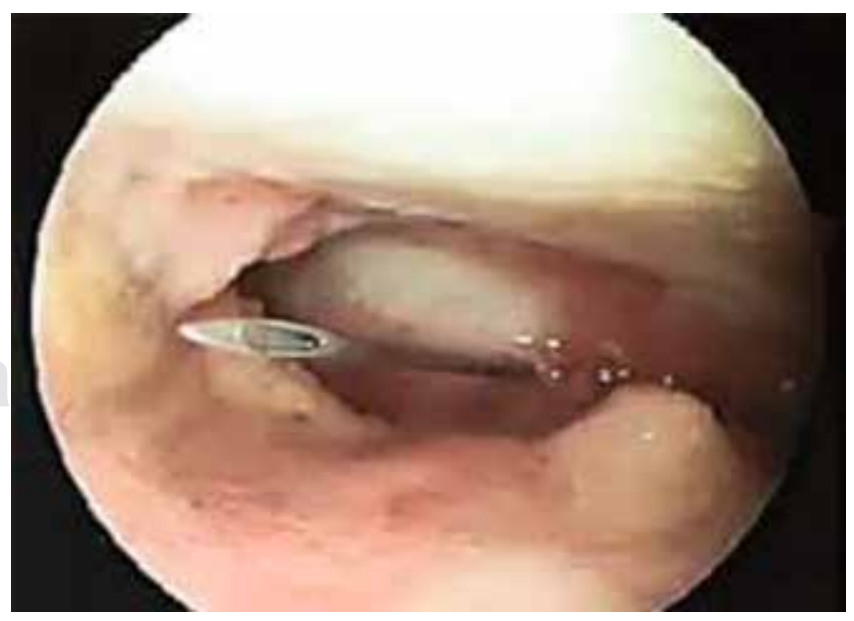

Figura 4: Infiltración epidérmica. 
Tabla 1: Variables clínicas según grupos.

\begin{tabular}{|c|c|c|c|c|c|c|c|}
\hline \multirow{3}{*}{\multicolumn{2}{|c|}{ Variables }} & \multicolumn{4}{|c|}{ Tipo de anestesia } & & \\
\hline & & \multicolumn{2}{|c|}{ Grupo AS } & \multicolumn{2}{|c|}{ Grupo IA } & \multicolumn{2}{|c|}{ Total } \\
\hline & & No & $\%$ & No & $\%$ & No & $\%$ \\
\hline \multirow{2}{*}{$\begin{array}{l}\text { Rodilla afectada } \\
(p=0.408)\end{array}$} & Derecha & 42 & 68.5 & 35 & 58.3 & 77 & \\
\hline & Izquierda & 18 & 30.0 & 25 & 41.7 & 43 & 35.8 \\
\hline \multicolumn{2}{|c|}{$M p$ interna $(p=0.253)$} & 25 & 41.7 & 18 & 30.0 & 43 & 35.8 \\
\hline \multicolumn{2}{|c|}{ Enf Hoffa $(p=0.717)$} & 5 & 8.3 & 3 & 5.0 & 8 & 6.7 \\
\hline \multicolumn{2}{|c|}{ Mp externa $(p=0.0 .688)$} & 16 & 26.7 & 19 & 31.7 & 35 & 29.2 \\
\hline \multicolumn{2}{|c|}{ Condromatosis $(p=0.0 .496)$} & 2 & 3.3 & 0 & 0.0 & 2 & 1.7 \\
\hline \multicolumn{2}{|c|}{ Lisis $(p=1.000)$} & 1 & 1.7 & 0 & 0.0 & 1 & 0.8 \\
\hline \multicolumn{2}{|c|}{ Queilectomía $(p=0.761)$} & 7 & 11.7 & 5 & 8.3 & 12 & 10.0 \\
\hline \multicolumn{2}{|c|}{ Plica $(p=1.000)$} & 35 & 58.3 & 36 & 60.0 & 71 & 59.2 \\
\hline \multicolumn{2}{|c|}{$\operatorname{SVP}(p=1.000)$} & 2 & 3.3 & 1 & 1.7 & 3 & 2.5 \\
\hline \multicolumn{2}{|c|}{$A R(p=0.361)$} & 4 & 6.7 & 8 & 13.3 & 12 & 10.0 \\
\hline \multicolumn{2}{|c|}{$\operatorname{EPDC}(p=1.000)$} & 14 & 23.3 & 13 & 21.7 & 27 & 22.5 \\
\hline \multicolumn{2}{|c|}{ Total } & \multicolumn{2}{|c|}{60} & \multicolumn{2}{|c|}{60} & \multicolumn{2}{|c|}{120} \\
\hline
\end{tabular}

Fuente: Hoja de recolección de datos.

*Prueba $x^{2}$. Abreviaturas: AS = Anestesia subaracnoidea. Al = Anestesia intraarticular con anestesia local. Mp Interna $=$ Meniscectomía parcial interna. Mp Externa $=$ Meniscectomía parcial externa. $\mathrm{SVP}=$ Sinovitis vellonodular pigmentada. $\mathrm{AR}=$ Artritis Reumatoide. $\mathrm{EPDC}=$ Enfermedad por depósitos de cristales.

\begin{tabular}{|c|c|c|c|c|c|c|}
\hline \multirow[b]{3}{*}{ Osteoartritis } & \multicolumn{4}{|c|}{ Tipo de anestesia } & & \\
\hline & \multicolumn{2}{|c|}{ Grupo AS } & \multicolumn{2}{|c|}{ Grupo IA } & \multicolumn{2}{|c|}{ Total } \\
\hline & No & $\%$ & No & $\%$ & No & $\%$ \\
\hline No & 28 & 46.7 & 30 & 50.0 & 58 & 48.3 \\
\hline Ligera & 4 & 6.7 & 5 & 8.3 & 9 & 7.5 \\
\hline Moderada & 2 & 3.3 & 1 & 1.7 & 3 & 2.5 \\
\hline Severa & 26 & 43.3 & 24 & 40.0 & 50 & 41.7 \\
\hline Total & \multicolumn{2}{|c|}{60} & \multicolumn{2}{|c|}{60} & \multicolumn{2}{|c|}{120} \\
\hline
\end{tabular}

Fuente: Hoja de recolección de datos.

complicaciones, mientras que en el Grupo IA sólo 15\% las tuvieron. Existieron diferencias significativas $(\mathrm{p}=0.049)$.

\section{DISCUSIÓN}

En esta serie, la media de edad que con mayor frecuencia se presentó fue en el grupo etario de entre 60-69 años, 28.3\% en el Grupo AS y 35.0\% en el Grupo IA, sin que existieran diferencias significativas $(\mathrm{p}=0.683)$. Se puede señalar que no existe consenso en la literatura con respecto a la frecuencia de edades ${ }^{(3,12,15,22-27,29-33)}$.

Aunque en la literatura revisada ${ }^{(22,25,34)}$ la artroscopía de rodilla predomina en el sexo masculino, en esta investiga- ción la mayor frecuencia estuvo en el sexo femenino (42/18 versus 44/16), en ambas técnicas anestésicas. No existieron diferencias significativas $(\mathrm{p}=0.839)$.

Moreno-Regidor y colaboradores ${ }^{(27)}$ plantearon que el desarrollo de las técnicas quirúrgicas poco invasivas, como la artroscopía y la mejora en el control de la analgesia postoperatoria mediante técnicas anestésicas locales o locorregionales, hicieron posible que el tiempo de estancia hospitalaria de los pacientes disminuyera, así como el desarrollo de programas de cirugía ambulatoria y de corta estancia que evitan gastos innecesarios. Desde que se realiza la artroscopía de rodilla con anestesia local ${ }^{(3)}$, el resto de las técnicas anestésicas se han relegado, ya que éstas no 
controlan el dolor postoperatorio y no permiten una rehabilitación temprana ${ }^{(34,35)}$.

La anestesia general, regional o troncular proporcionan una gran comodidad en el momento del proceder quirúrgico, pero provocan un bloqueo motor completo que imposibilita la rehabilitación precoz; con la anestesia general no existe suficiente analgesia postoperatoria, fundamental para la rehabilitación. Moreno-Regidor, Wallace y colaboradores ${ }^{34}$ y Wang y su equipo ${ }^{35}$ hacen referencia a las ventajas de la AIAR como método ideal para la artroscopía de la rodilla, sobre todo en aquéllos con régimen de cirugía ambulatoria, pues permite la realización de la técnica quirúrgica, disminuye el consumo de analgésicos para proporcionar una adecuada analgesia y una alta satisfacción. Asimismo, permite una pronta recuperación, un alta hospitalaria y una rehabilitación precoces ${ }^{(27,29,30)}$.
Según González y colegas( ${ }^{(29)}$, la cirugía artroscópica de rodilla realizada de manera ambulatoria, impone el reto de encontrar la técnica anestésica más adecuada para este tipo de procedimiento. Camponovo ${ }^{(30)}$ señaló que las mayores ventajas dependen de la selección correcta del anestésico local, pues en anestesia subaracnoidea constituye una técnica segura, de duración ultracorta para pacientes ambulatorios.

Wallace y su equipo ${ }^{(34)}$ concuerdan que el bloqueo de nervio periférico es un método muy popular para el tratamiento del dolor postoperatorio después de procedimientos quirúrgicos de miembros inferiores. Estos autores plantearon que, además del bloqueo intraarticular, se pueden utilizar bloqueos de nervios periféricos para artroscopía de rodilla que incluyen el bloqueo 3 en 1 y el bloqueo compartimental de la fascia ilíaca. Hay límites en la comparación de investigaciones de la eficacia de estos bloqueos en adultos para artroscopía de rodilla. Se com-

\begin{tabular}{|c|c|c|c|c|c|c|}
\hline \multirow{3}{*}{\multicolumn{2}{|c|}{ Evolución del dolor }} & \multicolumn{4}{|c|}{ Tipo de anestesia } & \multirow[b]{3}{*}{ Significación $(p)$} \\
\hline & & \multicolumn{2}{|c|}{ Grupo AS } & \multicolumn{2}{|c|}{ Grupo IA } & \\
\hline & & No & $\%$ & No & $\%$ & \\
\hline \multirow[t]{2}{*}{ Postoperatorio } & No & 47 & 78.3 & 54 & 90.0 & 0.1335 \\
\hline & Sí & 13 & 21.7 & 6 & 10.0 & \\
\hline \multirow[t]{2}{*}{3 horas } & No & 55 & 91.7 & 60 & 100 & $0.0573^{*}$ \\
\hline & Sí & 5 & 8.3 & 0 & 0.0 & \\
\hline \multirow[t]{2}{*}{6 horas } & No & 50 & 83.3 & 59 & 98.3 & 0.0114 \\
\hline & Sí & 10 & 16.7 & 1 & 1.7 & \\
\hline \multirow[t]{2}{*}{12 horas } & No & 43 & 71.7 & 51 & 85 & 0.1209 \\
\hline & Sí & 17 & 28.3 & 9 & 15 & \\
\hline \multirow[t]{2}{*}{24 horas } & No & 40 & 66.7 & 47 & 78.3 & 0.2199 \\
\hline & Sí & 20 & 33.3 & 13 & 21.7 & \\
\hline Total & & \multicolumn{2}{|c|}{60} & \multicolumn{2}{|c|}{60} & \\
\hline
\end{tabular}

\begin{tabular}{|c|c|c|c|c|c|c|}
\hline \multirow{2}{*}{\multicolumn{2}{|c|}{ Complicaciones }} & \multicolumn{2}{|c|}{ Grupo AS } & \multicolumn{2}{|c|}{ Grupo IA } & \multirow[b]{2}{*}{ Significación $(p)^{*}$} \\
\hline & & No & $\%$ & No & $\%$ & \\
\hline \multirow[t]{2}{*}{ Presencia } & No & 42 & 70.0 & 51 & 85.0 & 0.049 \\
\hline & Sí & 18 & 30.0 & 9 & 15.0 & \\
\hline \multirow[t]{4}{*}{ Tipo } & HTA & 8 & 44.4 & 3 & 33.3 & 0.788 \\
\hline & Hipotensión & 8 & 44.4 & 4 & 44.4 & 0.793 \\
\hline & Taquicardia & 1 & 5.6 & 1 & 11.1 & 1.000 \\
\hline & Bradicardia & 1 & 5.6 & 1 & 11.1 & 1.000 \\
\hline Total & & & & & & \\
\hline
\end{tabular}


probó que el inicio del bloqueo, el tiempo de requerimientos de analgesia postoperatorio, las escalas de evaluación del dolor y la satisfacción no mostraron diferencias significativas.

Wang y asociados ${ }^{(35)}$ concluyeron que cuando se compararon la anestesia epidural continua baja y la AIAR se obtuvieron mejores resultados con la AIAR, pues con anestesia local la condición de los nervios y de los vasos puede ser oportuna y observada de forma dinámica, con mejores ventajas, menor trauma, menor tasa de recurrencia y resultados más satisfactorios.

En esta investigación, al evaluar el bloqueo regional intraarticular con anestésicos locales, según la presencia de dolor, se pudo señalar que el dolor postoperatorio sólo estuvo presente en $21.7 \%$ del grupo AS y en $10 \%$ del Grupo IA. A las seis horas del postoperatorio, el dolor se presentó en 16.7 $\%$ del Grupo AS y en sólo 1.7\% del Grupo IA, en la que existieron diferencias significativas entre grupos $(p=0.0114)$.

Ravnihar y colaboradores ${ }^{(36)}$ realizaron un estudio retrospectivo donde demostraron que una inyección única de lidocaína utilizada para la artroscopía de rodilla no influyó en la viabilidad, la morfología y el potencial de cultivo de condrocitos en muestras de biopsia de cartílago articular asignados para la implantación de condrocitos autólogos con un nivel de evidencia IV.

Znojek-Tymborowska y su grupo ${ }^{(37)}$, a pesar de las diferencias, con relación al dolor postoperatorio y la movilidad de la rodilla, los resultados obtenidos a lo largo del período postoperatorio no permitieron favorecer ni a la anestesia infiltrativa intraarticular ni a la regional en la artroscopía de rodilla con un nivel de evidencia II.

Yari y colegas ${ }^{(28)}$ determinaron la mejor dosis única de morfina intraarticular para aliviar el dolor postcirugía artroscópica de rodilla y determinar la mejor analgesia a largo plazo, con menos efectos secundarios sistémicos. Para ello, administraron en 40 atletas ASA-I (después del procedimiento quirúrgico) una inyección de $20 \mathrm{~mL}$ de $0.5 \%$ intraarticular de bupivacaína con 5, 10 y $15 \mathrm{mg}$ de morfina. Los valores de la EVA en la cuarta, sexta y vigésima cuarta horas mostró diferencias significativas entre grupos. Los resultados de asociar bupivacaína con $15 \mathrm{mg}$ de morfina fueron significativamente mejores. La demanda de analgésicos fue significativamente menor que en otros grupos. Las complicaciones observadas fueron en las primeras 24 horas después de la inyección. Concluyeron que la aplicación de $15 \mathrm{mg}$ de morfina intraarticular después de la cirugía artroscópica de rodilla aumentó el nivel de analgesia, así como su duración.

Para identificar en esta muestra, la presencia de complicaciones según grupos, se puede señalar que en el Grupo AS se presentaron un total de $30.0 \%$ de complicaciones, mientras que en el Grupo IA sólo $15.0 \%$ con diferencias significativas $(\mathrm{p}=0.049)$. Dentro de ellas, las más frecuentes fueron: hipertensión arterial (15.0 y 11.0\% según grupos) e hipotensión arterial (15.0 y 13.3\% según grupo) en el Grupo AS, mientras en el AI primó el dolor.

Shaukat y su equipo ${ }^{(38)}$ concluyeron que la anestesia local intraarticular en artroscopía de rodilla constituye una contundente alternativa a otras formas de técnicas anestésicas y además debe ser considerada como el estándar de oro en este tipo de cirugías.

Se concluye que los resultados del bloqueo regional intraarticular con anestésicos locales en la cirugía artroscópica de rodilla constituye una técnica fácil y segura con escasas complicaciones. El uso de bloqueos guiados por ultrasonidos es una realidad en este momento, que modificará estas técnicas.

\section{REFERENCIAS}

1. Mondino JA. Artroscopia de rodilla con anestesia local. Rev Arg Artroscopia. 2002;12:102-110.

2. Diéguez-García P, López-Álvarez S, Blanco-Dávila R, Rebollo-Laserna J, Zaballos-Bustingorri E, Monzó-Abad E, et al. Estudio epidemiológico multicéntrico de las técnicas anestésicas en la cirugía de la artroscopia de rodilla en España. Cir May Amb. 2012;17:25-32.

3. Williams BA, Matusic B, Kentor ML. Regional anesthesia procedures for ambulatory knee surgery: Effects on in-hospital outcomes. Int Anesthesiol Clin. 2005;43:153-160.

4. Williams BA, Kentor ML, Williams JP, Vogt MT, DaPos SV, Harner CD, et al. PACU bypass after outpatient knee surgery is associated with fewer unplanned hospital admissions but more phase II nursing interventions. Anesthesiology. 2002;97:981-988.

5. Zaballos M, López-Alvarez S, Zaballos-Bustingorri J, Rebollo-Laserna F, De La Pinta JC, Monzó-Abad E. Estudio epidemiológico multicéntrico de las técnicas anestésicas en la cirugía de la hernia inguinal en España. Rev Esp Anestesiol Reanim- 2012;59:18-24.

6. Memtsoudis S, Kuo C, Ma Y, Edwards A, Mazumdar M, Liguori G. Changes in Anesthesia-related factors in ambulatory knee and shoulder surgery. United States 1996-2006. Reg Anesth Pain Med. 2011;36:327-331.
7. Korhonen A, Valanne J, Jokela R, Ravaska P, Korttila K. A comparison of selective spinal anesthesia with hyperbaric bupivacaine and general anesthesia with desflurane for outpatient knee arthroscopy. Anesth Analg. 2004;99:1668-16673.

8. Korhonen AM. Use of spinal anaesthesia in day surgery. Curr Opin Anaesthesiol. 2006;19:612-616.

9. Ben-David B, Levin H, Solomon E, Admoni H, Vaida S. Spinal bupivacaine in ambulatory surgery: the effect of saline dilution. Anesth Analg. 1996;83:716-720.

10. Borghi B, Stagni F, Bugamelli S, et al. Unilateral spinal block for outpatient knee arthroscopy: a dose-finding study. J Clin Anesth. 2003;15:351-356.

11. Kiran S, Upma B. Use of small-dose bupivacaine (3 mg vs. $4 \mathrm{mg}$ ) for unilateral spinal anesthesia in the outpatient setting. Anesth Analg. 2004;99:302-303.

12. Nair G.S, Abrishami A, Lermitte J, Chung F. Systematic review of spinal anaesthesia using bupivacaine for ambulatory knee arthroscopy. Br J Anaesth. 2009;102:307-315.

13. Fanelli G, Borghi B, Casati A, Bertini L, Montebugnoli M, Torri G. Unilateral bupivacaine spinal anesthesia for outpatient knee arthroscopy. 
Italian Study Group on Unilateral Spinal Anesthesia. Can J Anaesth. 2000;47:746-751.

14. Esmaoglu A, Karaoglu S, Mizrak A, Boyaci A. Bilateral vs. unilateral spinal anesthesia for outpatient knee arthroscopies. Knee Surg Sports Traumatol Arthrosc. 2004;12:155-158.

15. Liu SS, Strodtbeck WM, Richman JM, Wu CL. A comparison of regional versus general anesthesia for ambulatory anesthesia: A metaanalysis of randomized controlled trials. Anesth Analg. 2005;101:16341642.

16. Hadzic A, Karaca P, Hobeika P, Unis G, Dermksian J, Yufa M, et al. Peripheral nerve blocks result in superior recovery profile compared with general anesthesia in outpatient knee arthroscopy. Anesth Analg. 2005;100:976-981.

17. Elvir-Lazo OL, White PF. Postoperative pain management after ambulatory surgery: Role of multimodal analgesia. Anesthesiol Clin. 2010;28:217-224.

18. Miskulin M, Maldini B. Outpatient arthroscopic knee surgery under multimodal analgesic regimens. Arthroscopy. 2006;22:978-983.

19. Atef H, El-Kasaby Ael-D, Omera M, Badr M. Optimal dose of hyperbaric bupivacaine $0.5 \%$ for unilateral spinal anesthesia during diagnostic knee arthroscopy. Middle East J Anesthesiol. 2012;21:591-598.

20. Wallace JB, Andrade JA, Christensen JP, Osborne LA, Pellegrini JE. Comparison of fascia iliaca compartment block and 3-in-1 block in adults undergoing knee arthroscopy and meniscal repair. AANA J. 2012;80:S37-44

21. Knautz J, Asher Y, Kendall MC, Doty R Jr. Ambulatory anesthesia in an adult patient with corrected hypoplastic left heart syndrome. Case Rep Anesthesiol. 2012;2012:607140.

22. Adekoya-Cole TO, Enweluzo GO, Akinmokun OI, Orakwe DE. Basic arthroscopy: a review paper. Nig Q J Hosp Med. 2011;21:303-305.

23. Da Assunção RE, Neely J, Lochab J, Mizumi-Richards N, Barnett A, Pandit H. Patient recall of surgical information after day case knee arthroscopy. Knee Surg Sports Traumatol Arthrosc. 2012;2:228-232.

24. Unal D, Ozdogan L, Ornek HD, Sonmez HK, Ayderen T, Arslan M, Dikmen B. Selective spinal anaesthesia with low-dose bupivacaine and bupivacaine + fentanyl in ambulatory arthroscopic knee surgery. J Pak Med Assoc. 2012;62:313-318.

25. Goëb V, Walsh CA, Reece RJ, Emery P, Ponchel F. Potential role of arthroscopy in the management of inflammatory arthritis. Clin Exp Rheumatol. 2012;30:429-435.
26. Yera Nadal JL, Guillén Vargas M, Squire Valdés E, Rodríguez Valdés M, Hernández Luaces LF. Analgesia postoperatoria en la cirugía artroscópica de rodilla. Estudio multicéntrico. Rev Cubana Anest Rean. 2003;2:31-36.

27. Moreno-Regidor A, Yusta-Martín G, Borrego-Ratero D, Blanco-Blanco, J. Anestesia local para artroscopia de rodilla en pacientes ambulatorios. Rev Ortp Traumatol. 2007;51:39-41.

28. Yari M, Saeb M, Golfam P, Makhloogh Z. Analgesic efficacy of intraarticular morphine after arthroscopic knee surgery in sport injury patients. J Inj Violence Res. 2013;5:84-88.

29. González O MP, Vásquez Sadder M I, Herrera Morales LA, Rivera DRC, Arcila L MA, Puerta M JJ Trujillo H LM; Torres de Galvis Y. Anestesia general versus regional para cirugía artroscópica ambulatoria de rodilla. CES Medicina. 2009;23:7-16.

30. Camponovo C. Spinal 1\% 2-Chloroprocaine versus general anesthesia for ultra-short outpatient procedures: a retrospective analysis. Acta Biomed. 2014;85:265-268.

31. Guntz E, Latrech B, Tsiberidis C, Gouwy J, Kapessidou Y. ED50 and ED90 of intrathecal hyperbaric $2 \%$ prilocaine in ambulatory knee arthroscopy. Can J Anaesth. 2014;61:801-807.

32. Lakhin RE, Schegolev AV, Panov VA, Kuligin AV. Low dose spinal anesthesia for knee arthroscopy. Anaesth Reanimatol. 2015;11:126-128.

33. Bergmann I, Hesjedal B, Crozier TA, Pöschl R, Bauer M, Hinz JM, Büttner B. Selective unilateral spinal anaesthesia for outpatient knee arthroscopy using real-time monitoring of lower limb sympathetic tone. Anaesth Intensive Care. 2015;43:351-356.

34. Wallace JB, Andrade JA, Christensen JP, Osborne LA, Pellegrini JE. Comparison of fascia iliaca compartment block and 3-in-1 block in adults undergoing knee arthroscopy and meniscal repair. AANAJ. 2012;80:S37-44.

35. Wang J, Liu Y, Wang J, Qi W, Qu F, Shen X et al. Arthroscopic treatment of popliteal cyst excision in combination with debridement of the knee under local anesthesia. Zhongguo Xiu Fu Chong Jian Wai Ke Za Zhi. 2014;28:933-937.

36. Ravnihar K, Barlič A, Drobnič M. Effect of intra-articular local anesthesia on articular cartilage in the knee. Arthroscopy. 2014;30:607-612.

37. Znojek-Tymborowska J, Kęska R, Paradowski PT, Witoński D. Relevance of infiltration analgesia in pain relief after total knee arthroplasty. Acta Ortop Bras. 2013;21:262-265.

38. Shaukat Y, Malik E, El-Khateeb H, Koeweiden E. The role of local anaesthesia in knee arthroscopy. J Orthop. 2013;10:193-195. 